\title{
Thermal regimes and THz generation from BSCCO mesas
}

\author{
L.S. Revin ${ }^{1}$, E.A. Vopilkin ${ }^{1}$, A.L. Pankratov ${ }^{1}$, S.A. Kraev ${ }^{1}$, \\ A.A. Yablokov ${ }^{1}$, S.A. Churin ${ }^{1}$, A.B. Kulakov ${ }^{2}$ \\ ${ }^{1}$ Institute for Physics of Microstructures of Russian Academy of Sciences, Nizhny Novgorod, Russia, alp@ipmras.ru \\ ${ }^{2}$ Institute of Solid State Physics of RAS
}

At present, the question of creating generators of subTHz and $\mathrm{THz}$ ranges based on $\mathrm{Bi}_{2} \mathrm{Sr}_{2} \mathrm{CaCu}_{2} \mathrm{O}_{8+\mathrm{x}}$ (BSCCO) mesastructures is of great interest [1]. BSCCO is a high-temperature superconductor with strong anisotropy, which leads to the appearance of an internal Josephson effect [2]. In other words, $\mathrm{CuO}$ layers represent a series of Josephson chains formed on an atomic scale. The first success in observing of electromagnetic radiation from BSCCO structures was achieved in 2007 [3]. The observed radiation had a power of the order of $0.5 \mu \mathrm{W}$ and a frequency up to $0.85 \mathrm{THz}$. Since that time the radiation power has been increased to a value of hundreds of microwatts [4]. These achievements make it possible to use BSCCO mesostructures as sources for noise spectroscopy $[5,6]$.

In our group the technology of electrolytic buildup of a BSCCO/Cu heterostructures is worked out, representing copper covering over a thin layer of gold, that allows to get rigid single BSCCO mesastructures with a good heatsink. To obtain the described samples, we used a BSCCO single crystal, fabricated at ISSP RAS, of several microns thick with lateral dimensions of several millimeters. On one of its surfaces, a layer of $50 \mathrm{~nm}$ thick gold was deposited by thermal deposition. After this a layer of copper with thickness of about 30 microns was electrolytically built up on top of the gold layer. Then a layer of 50 nm thick gold was deposited on BSSCO second surface by thermal deposition. After that, using the original method of fast chemical etching [7], developed in our team, the cylindrical single mesastructures were fabricated. The obtained sample was glued to the copper holder, Fig. 1, after which it is taped to the contact pads and installed in a cryostat.

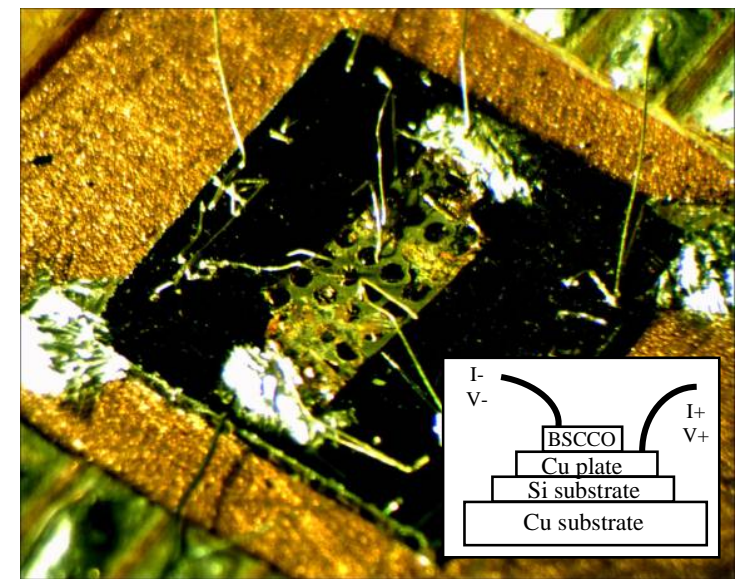

Fig. 1. Photo of the mesas connected by wires. Inset: Schematic view of the Si substrate with BSCCO sample, attached to the copper holder
To measure the radiated power, the room temperature detector (Golay cell) together with the horn and infrared filters were used, Fig. 2. Although the obtained samples were not intended for effective power radiation, since antennas and lenses were not supposed, the level of the expected signal was sufficient. The calculated diagram had maxima at $45^{\circ}$ from the plane and only a small amount of radiation was detected outside the cryostat window with the holder parallel to the cryostat plate.

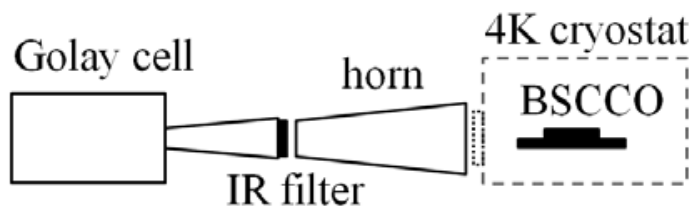

Fig. 2. Schematic of the experimental setup

In Fig. 3 the current-voltage characteristic of BSCCO mesa is presented, forward and backward branches are measured. The shape of the curve indicates the presence of Josephson generation in the structure. On the forward branch there are transitions from the superconducting state to the resistive one both of the individual junctions of the layered superconductor and of the entire structure. On the backward branch there is an area of negative differential resistance, which indicates the presence of a "hot spot" inside the mesastructure, the heating region of the structure, which is the channel for synchronization of electromagnetic waves in the superconductorinsulator-superconductor layers.

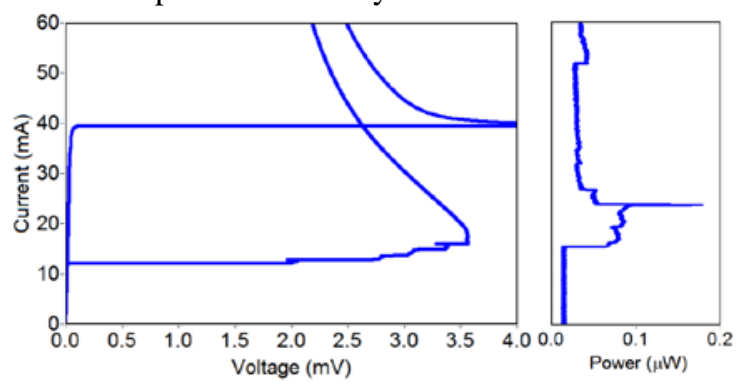

Fig. 3. The voltage and received power as a function of bias current

As a result, the statistics of the obtained structures were processed, and the characteristics of the samples were compared with the world level. Thus, the critical current density of the obtained samples $J_{\mathrm{c}}=30-70$ $\mathrm{A} / \mathrm{cm}^{2}$ is close to the literature data $J_{\mathrm{c}}=80-250$ $\mathrm{A} / \mathrm{cm}^{2}$. The gap voltage in terms of one junction $V_{\mathrm{g}}=$ 
$0.6-0.7 \mathrm{mV}$ is also close to the literature data $V_{\mathrm{g}}=1$ $1.4 \mathrm{mV}$.

Experiments on detecting the radiation from BSCCO sample allow to obtain a signal of the order 0.05-0.08 $\mu \mathrm{W}$. Radiation was observed at the plate temperature of $18 \mathrm{~K}$ when the substrate was attached to a copper holder by a rubber glue. Conversely, when the substrate was attached by indium, no radiation was observed, presumably due to a too-low holder temperature at a better heat sink or not enough overheating due to a too-efficient heat sink.

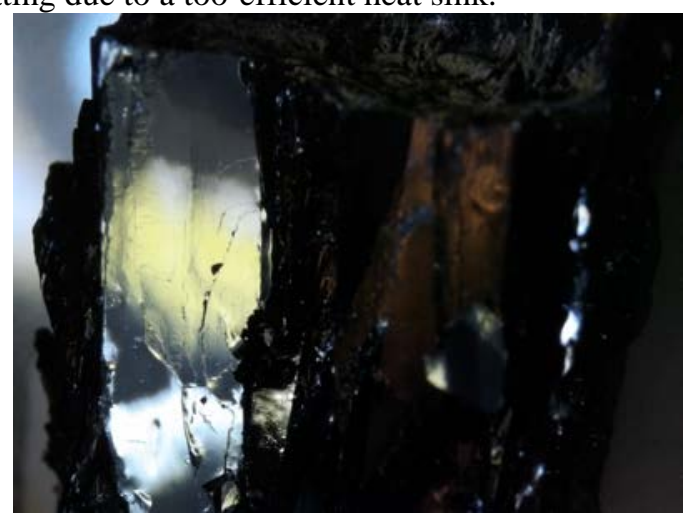

Fig. 4. Photo of BSCCO single crystal fabricated by Bridgman technique

Recently, first BSCCO single crystal samples have been fabricated at IPM RAS by Bridgman technique, having a plain surface of order 3 by $10 \mathrm{~mm}$, see Fig. 4. The samples have been measured in a cryostat and critical temperature of $84 \mathrm{~K}$ has been achieved for oxigen annealed samples, see Fig. 5. Currently, new BSCCO single mesas with $\mathrm{Cu}$ sublayer from these single crystals are under fabrication.

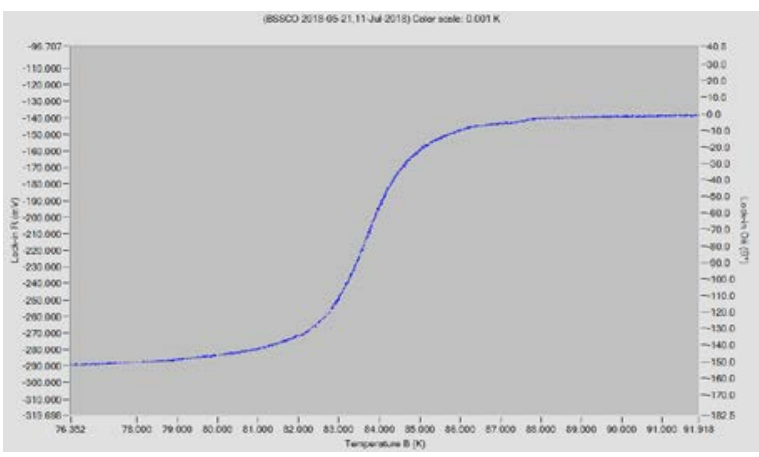

Fig. 5. Measurement of BSCCO single crystal critical temperature
In conclusion, we report on the progress of the wet etching method for the fabrication of BSCCO mesastructures, making it possible to obtain samples with a large thickness on the order of $1-15 \mu \mathrm{m}$. The current-voltage curves of BSCCO mesas are measured at different plate temperatures and there is a significant dependence on the thermal interface. It is possible to determine the power (of the order of 0.1 $\mu \mathrm{W}$ ) outside the cryostat by a room temperature detector. BSCCO single crystals, having critical temperature of $84 \mathrm{~K}$ were fabricated by Bridgman technique.

The work is supported by RSF (project 16-1910478).

\section{References}

1. Welp, U., Kadowaki, K., Kleiner, R., Superconducting emitters of THz radiation // Nat. Photonics. 2013, Vol. 7, pp. 702

2. Kleiner R.,, Steinmeyer, F., Kunkel G., Mueller P., Intrinsic Josephson effects in $\mathrm{Bi} 2 \mathrm{Sr} 2 \mathrm{CaCu} 2 \mathrm{O} 8$ single crystals // Phys. Rev. Lett. 1992, Vol. 68, p. 2394.

3. Ozyuzer, L., Koshelev, A.E., Kurter, C., Gopalsami, $N$., et al Emission of Coherent THz Radiation from Superconductors // Science 318, 2007, p. 1291.

4. Yamaki, K., Tsujimoto, M., Yamamoto, T., Furukawa, A., Kashiwagi, T., Minami, H., Kadowaki, K., Highpower terahertz electromagnetic wave emission from high- $\mathrm{T}$ c superconducting $\mathrm{Bi} 2 \mathrm{Sr} 2 \mathrm{CaCu} 2 \mathrm{O} 8+\delta$ mesa structures // Opt. Express. 2011. Vol. 19, P. 3193.

5. Revin, L.S., Vaks, V.L., Koshelets, V.P., Wang, H., // EPJ Web of Conferences. 2017, Vol. 132, P. 03042.

6. Sobakinskàya, E.A., Vax, V.L., Kinev, N.V., Koshelets, V.P., Wang, H., // Proc. 1st Russian Microwave Conf. 2013, p. 1015.

7. Vopilkin E.A., Chiginev A.V., Revin L.S., Tropanova A.N., Shuleshova I.Yu., Okhapkin A.I., Shovkun A.D., Kulakov A.B., Pankratov A.L., Quick and reliable technology for fabrication of stand-alone BSCCO mesas, // Supercond. Sci. Technol. 2015, Vol. 28, P. 045006. 